\title{
Probe wave system for studying excitation of Alfvén eigenmodes in the ion cyclotron range of frequency
}

\author{
M. Ichimura, ${ }^{\text {a) }}$ Y. Ohta, S. Motegi, M. Nakamura, S. Tanaka, S. Kanazawa, S. Saosaki, \\ C. Nakagawa, K. Sakata, K. Kadoya, T. Kawabata, M. Oikawa, H. Kano, M. Sasaki, \\ H. Hojo, and K. Yatsu \\ Plasma Research Center, University of Tsukuba, Tsukuba, Ibaraki 305-8577, Japan
}

(Presented on 19 June 2000)

\begin{abstract}
A probe wave system was constructed for studying the excitation of the Alfvén ion cyclotron (AIC) modes in the anchor cell of the GAMMA 10 tandem mirror. In the central cell, the AIC modes are spontaneously excited as axial eigenmodes due to the strong temperature anisotropy. In the anchor cell, the AIC modes were not observed though the anisotropy is the same as in the central cell. The axial profiles of the magnetic field strength and plasma parameters in the anchor cell are different from those in the central cell. Eigenmodes are excited externally in the AIC frequency range by injecting probe waves. This suggests the AIC modes are unstable in the anchor cell and grow to the detectable level when boundary conditions are realized. (c) 2001 American Institute of Physics.

[DOI: $10.1063 / 1.1315638$ ]
\end{abstract}

\section{INTRODUCTION}

When the wavelength of the modes in the ion cyclotron range of frequency (ICRF) is in the same order of the plasma size, the excitation and propagation of the waves are strongly affected by the boundary conditions. The boundary conditions are determined by the characteristic length of the spatial variations of the magnetic field strength and the plasma parameters, for example, the nonuniformity of the plasma density and so on. Recently, the toroidicity induced Alfvén eigenmodes have been excited externally in the Joint European Torus. ${ }^{1}$ From the spectroscopic view, discrete Alfvén waves have been used for studying the magnetohydrodynamic (MHD) behavior in tokamaks ${ }^{2}$ and the propagation of ICRF waves are investigated in the Tokamak Fusion Test Reactor. ${ }^{3}$ In the GAMMA 10 tandem mirror, the ion temperature of the central cell reaches several kiloelectron volts and the Alfvén ion cyclotron (AIC) modes are spontaneously excited due to the strong temperature anisotropy. ${ }^{4}$ These modes are detected as axial eigenmodes determined from the plasma pressure profile in the axial direction. ${ }^{5}$ The condition predicted by a theory ${ }^{6}$ for the absolute instability is about one order of magnitude higher than that observed in the experiments. ${ }^{7}$ The mechanism for the excitation of AIC modes in the GAMMA 10 plasma is not clear at present. The plasma parameters in the anchor cell of the GAMMA 10 are almost the same as those in the central cell. However, the AIC modes in the anchor cell were not observed. The magnetic field configuration in the anchor cell is the minimum-B field and is different from that in the central cell. Because the formation of the eigenmodes is sensitive to the excitation of the Alfvén waves, the differences of the magnetic field and plasma parameter profiles in the axial direction should be important for the excitation of the AIC modes. In this article, the probe wave system to study the boundary conditions for

${ }^{a)}$ Electronic mail: ichimura@prc.tsukuba.ac.jp the excitation of the AIC modes is reported. By injecting low power ICRF waves into the anchor cell of the GAMMA 10, we measure the antenna response due to the excitation of the waves and waves excited in the plasma by using a magnetic probe.

\section{EXPERIMENTAL SETUP}

GAMMA 10 is a minimum-B anchored tandem mirror with axisymmetric plug/barrier cells at both ends. The central cell has an axisymmetric mirror field and is $5.6 \mathrm{~m}$ in length with a field strength of $0.4 \mathrm{~T}$ at the midplane. The mirror ratio of the central cell is 5 . The diameter of the stainless steel limiter which is installed near the midplane of the central cell is $0.36 \mathrm{~m}$. Two ICRF sources (RF1 and RF2) are used for the plasma production and heating. The plasma is started by applying ICRF pulses (RF1) of $9.9 \mathrm{MHz}$ (east) and $10.3 \mathrm{MHz}$ (west) with short pulse (1 ms) plasma guns at both ends and is sustained with hydrogen gas puffings. To avoid the strong interference between east RF1 and west RF1 antennas, the frequency of the west antenna is slightly higher than the frequency of the east antenna. These ICRF waves propagate to the anchor cell and heat ions in the cyclotron resonance layer near the midplane. The MHD stability of the GAMMA 10 plasma is kept by those high temperature ions. So-called Nagoya type III antennas are installed near both ends of the central cell. For the central cell ion heating, the ICRF pulse (RF2) of which a frequency of $6.36 \mathrm{MHz}$ is also used. Two heating antennas which are conventional double half-turn type antenna. The half-turn type antenna with a single layer Faraday shield is equipped on the inner side of the Nagoya Type III antenna. The ICRF power coupled to the plasma is about $100 \mathrm{~kW}$ for each RF system. Because the cyclotron resonance layer exists near the midplane, the strong ion heating and the resultant temperature anisotropy which is defined as an ion temperature ratio between perpendicular and parallel to the magnetic field line are observed. 


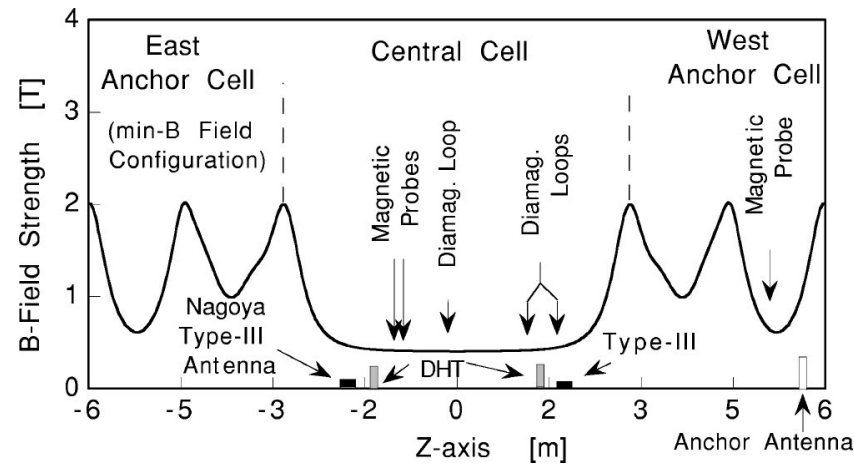

FIG. 1. The axial profile of the magnetic field strength in the central and anchor cells, and locations of ICRF antennas and diagnostics.

The temperature anisotropy is evaluated from a diamagnetic loop array. ${ }^{8}$ The AIC modes are spontaneously excited in a hot plasma with a strong temperature anisotropy. The anchor cells have a minimum-B mirror field configuration and are $1.6 \mathrm{~m}$ in length with a field strength of $0.6 \mathrm{~T}$ at the midplane and the mirror ratio is 3.3. As mentioned previously, the ions in the anchor cells are heated by RF1. To measure magnetic fluctuations, magnetic probes are set in the peripheral region of the plasma. The axial profile of the magnetic field strength in the central and anchor cells and locations of ICRF antennas and diagnostics are shown in Fig. 1. It is clearly shown that there is a big difference between the magnetic field profiles in the central cell and anchor cell.

\section{PROBE WAVE SYSTEM}

For studying the plasma response, we constructed a probe wave system in the AIC frequency range of the anchor plasma. Figure 2 shows a schematic diagram of the experimental arrangement of the probe wave system. The rf signals from a standard signal generator in the power level of milliwatts are mixed with the output of a pulse generator, which determines the pulse width and the trigger for the injection. The resultant rf pulse is amplified to several hundred watts and is applied to the antenna installed in the anchor cell $(z$ $=550 \mathrm{~cm}$ ) via a matching network. The antenna is a bar type antenna set on a quarter section of the plasma surface. The incident power of the amplifier and the reflected power are monitored using a bidirectional coupler set near the outlet of the amplifier. The current flowing on the antenna is detected by a small pickup coil. The waves excited in the plasma are detected by a magnetic probe located at $z=506 \mathrm{~cm}$. Because the position of the anchor midplane is $z=520 \mathrm{~cm}$, the antenna and the magnetic probe are set with an anchor midplane in between them. The frequency range of the injected rf signal is from 6.2 to $9.2 \mathrm{MHz}$. In this range, it is not necessary to change the matching parameters because the matching network is arranged for a relatively wide range of frequencies. The variation of the incident and reflected powers in a swept frequency mode without plasma are shown in Fig. 3. The frequency is also indicated in the figure. The frequency is swept in the stepwise as shown in Fig. 3. The frequency bandwidth is restricted by the time requirement needed for the fast Fourier transform analysis. The reflected power is kept low during the swept frequency mode and the

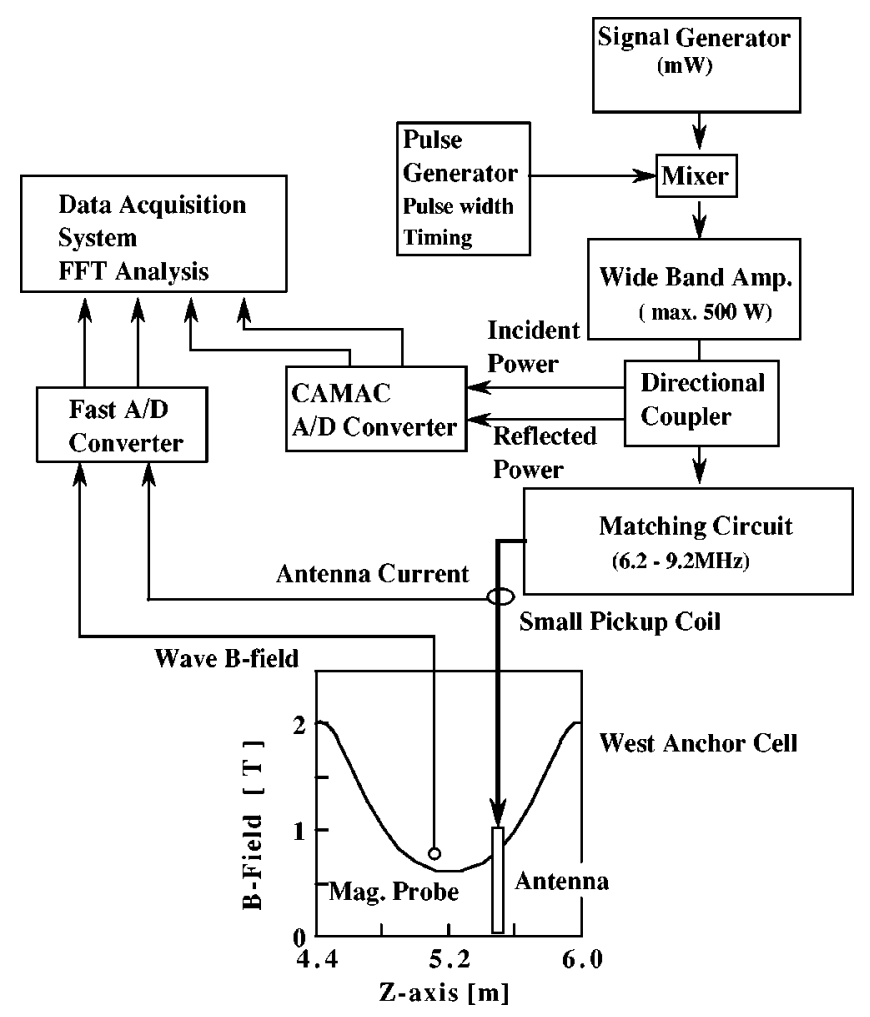

FIG. 2. A schematic diagram of the probe wave system.

matching condition is good in this frequency range. To investigate the plasma parameter dependence of the wave excitation, we can use the system in a fixed frequency mode during the discharge. When the spatial resonance exists in the plasma, for example, the eigenmode excitation of the AIC mode, the analysis of the response in the antennaplasma-probe transfer function will be effective. ${ }^{1}$ In the next section, the preliminary results in the fixed frequency mode are described.

\section{PRELIMINARY RESULTS AND DISCUSSIONS}

In this experiment, the frequency of the injected waves was fixed during each plasma discharge. The signals of the magnetic probe with and without the injection of the probe wave are shown in Fig. 4. On this discharge, the AIC modes are spontaneously excited due to the strong temperature anisotropy in the central cell. Small peaks just below a peak of $6.36 \mathrm{MHz}$ in the figure are the AIC modes excited in the

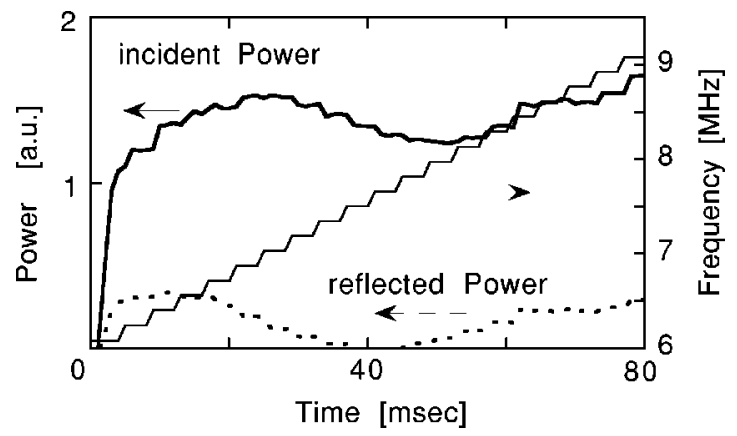

FIG. 3. Incident power of the amplifier and reflected powers in the swept frequency mode, the frequency is indicated in the figure. 

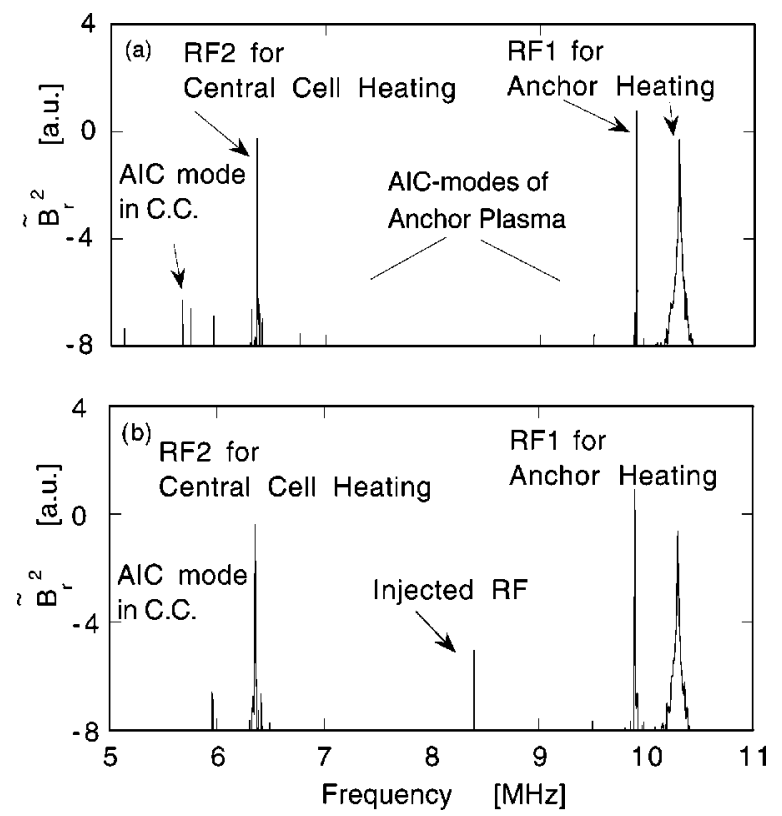

FIG. 4. Frequency spectrum of the magnetic probe signal (a) without and (b) with probe waves.

central cell propagating towards the anchor cell. The cyclotron frequency in the midplane of the anchor cell is $9.3 \mathrm{MHz}$. If the AIC modes are excited in the anchor cell, it is suggested that the signals will be observed near $0.85-0.95$ times ion cyclotron frequency as indicated in the figure. There are no clear peaks without the probe wave as shown in Fig. 4(a). When the waves are injected, a clear peak is observed as shown in Fig. 4(b). Figure 5(a) shows the temporal evolution of the line density of the anchor cell, and Fig. 5(b) shows the magnetic probe signals normalized by the radiated power as a function of the frequency of the injected waves. The magnetic probe signals depend on the injected frequency and peak around $30 \mathrm{~ms}$ whereas the line density in the anchor
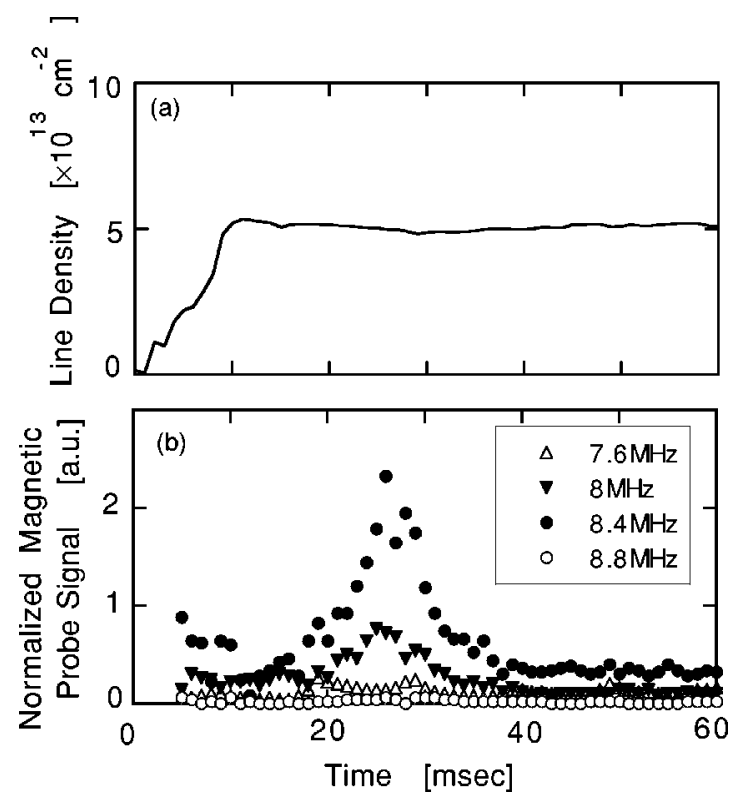

FIG. 5. Temporal evolution of (a) the line density in the anchor cell and (b) the magnetic probe signals normalized by the radiated power as functions of the injection frequency.

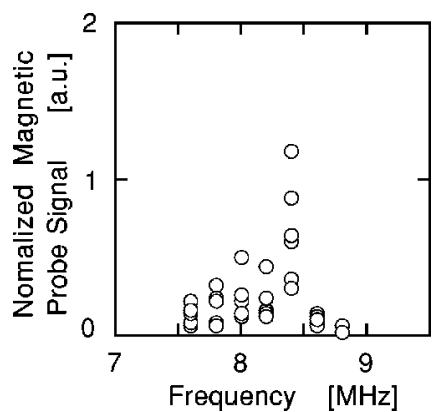

FIG. 6. Frequency dependence of the magnetic probe signals normalized by the radiated power.

cell is almost constant. The excitation of the waves will depend on the plasma parameters besides the density. The physical mechanism of these temporal evolution in unknown at present. Figure 6 shows the frequency dependence of the amplitude of the excited waves power at $30 \mathrm{~ms}$ which is also normalized by the radiated power. It is shown that the waves are resonantly excited near $8.4 \mathrm{MHz}$. As we described previously, this frequency is included in the unstable region of the AIC modes. This suggests the AIC modes are excited by the externally injected ICRF power in the anchor cell.

Figure 7 shows the typical frequency spectrum of the magnetic probe signal in the central cell. In the frequency spectrum there are several discrete peaks as shown in Fig. 7. The magnetic field components of these peaks have the same spatial profiles in the radial and azimuthal directions. ${ }^{9}$ The azimuthal mode number is $m=-1$. The axial structure was investigated precisely in Ref. 10. The AIC modes are excited as eigenmodes in the axial direction and have a standing wave structure in which the boundary conditions are determined by the plasma pressure. ${ }^{9}$ In the anchor cell, the conditions for the standing wave formation will be not established in the present parameter region. The preliminary results suggest the AIC modes can be excited externally by injecting the rf pulse. Because the axial profile of the magnetic field in the anchor cell is different from that in the central cell, there will be large differences between the conditions in the central cell and anchor cell.

\section{SUMMARY}

The excitation and propagation of ICRF waves are sensitive to boundary condition, for example, the spatial variation of the magnetic field strength, plasma parameters, and so

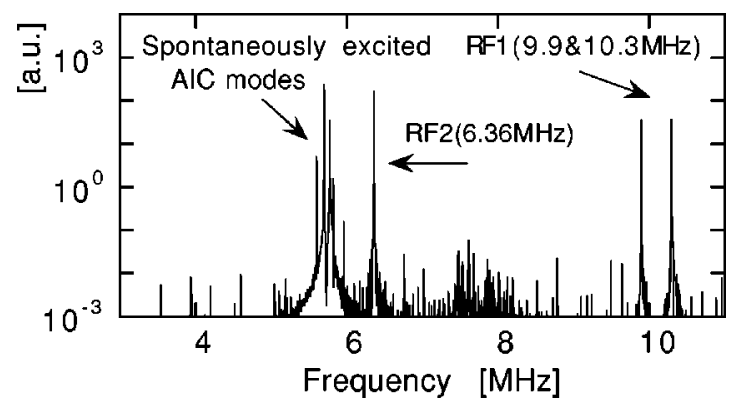

FIG. 7. Typical frequency spectrum of a magnetic probe signal in the central cell. 
on. A probe wave system was constructed for studying the excitation of the Alfvén waves in the GAMMA 10 tandem mirror.

In the central cell of the GAMMA 10, the AIC modes are spontaneously excited as axial eigenmodes due to the strong temperature anisotropy. However, in the anchor cell, the AIC modes were not observed though the anisotropy is the same as in the central cell. The axial profiles of the magnetic field strength and plasma parameters in the anchor cell are different from those in the central cell. Eigenmodes are excited externally in the AIC frequency range by injecting probe waves. This suggests that the AIC modes are unstable in the anchor cell and grow to the detectable level when boundary conditions are realized.

\section{ACKNOWLEDGMENTS}

The authors acknowledge the GAMMA 10 group of the University of Tsukuba for their collaboration. This work was supported in part by a Grant-in-Aid for Scientific Research from the Japanese Ministry of Education Science, Sports, and Culture.

\footnotetext{
${ }^{1}$ A. Fasoli et al., in Plasma Physics and Controlled Nuclear Fusion Research, Vol. I (IAEA, Vienna, 1993), p. 405.

${ }^{2}$ D. G. Miljak and R. C . Cross, Plasma Phys. Controlled Fusion 38, 207 (1996).

${ }^{3}$ G. J. Greene, Nucl. Fusion 35, 5831 (1995).

${ }^{4}$ M. Ichimura et al., Plasma Phys. Controlled Fusion 34, 1889 (1992).

${ }^{5}$ M. Nakamura et al., J. Plasma Fusion Res. 2, 314 (1999).

${ }^{6}$ G. R. Smith, T. A. Casper, and M. J. Gerver, Nucl. Fusion 23, 1381 (1983).

${ }^{7}$ M. Ichimura, M. Inutake, R. Katsumata, N. Hino, H. Hojo, K. Ishii, T. Tamano, and S. Miyoshi, Phys. Rev. Lett. 70, 2734 (1993).

${ }^{8}$ R. Katsumata et al., Jpn. J. Appl. Phys., Part 1 31, 2249 (1992).

${ }^{9}$ R. Katsumata, M. Ichimura, M. Inutake, H. Hojo, A. Mase, and T. Tamano, Phys. Plasmas 3, 4489 (1996).

${ }^{10}$ M. Nakamura et al., Rev. Sci. Instrum. (these proceedings).
} 